\title{
Effect of Ultrasonic-Assisted Extraction on Phenolic Content of Freshwater Macroalgae in Northern Thailand
}

\author{
Thitiphan Chimsook ${ }^{1, a}$ and Waranya Wannalangka ${ }^{1}$ \\ ${ }^{1}$ Faculty of Science, Department of Chemistry, Applied Chemistry, Maejo University, Chiang Mai, Thailand
}

\begin{abstract}
Effect of ultrasonic extraction on total phenolic content (TPC) of two algae; Spirogyra sp. and Cladophora glomerata was evaluated. In this study, the solid/solvent ratio of $1 / 30(\mathrm{wt} / \mathrm{vol})$ and extraction temperature of $40^{\circ} \mathrm{C}$ gave higher TPC value in two algae. This ratio and temperature was applied in the ultrasonic-assisted extraction (UAE) of phenolic extraction. This study compared the TPC obtained from two algae extracts without involving ultrasonic and the TPC obtained from the UAE. The results showed that the TPCs value of two algae was significantly higher in the UAE compared to the TPCs in the non-UAE. The increasing in the TPCs of two algae was between $\sim 23 \%$ and $\sim 39 \%$ when 5 to 20 min of ultrasonication applied in the extraction. Ultrasonication duration of 15 min and $10 \mathrm{~min}$ in Spirogyra sp. and Cladophora glomerata gave the highest TPCs which the value was significantly higher compared to the other duration.
\end{abstract}

\section{Introduction}

In general, medicinal herbs and dietary plants are a good resource of bioactive phenolic compounds. Phenolic compounds are ubiquitous and rich in medicinal herbs and dietary plants (e.g., fruits, vegetables, spices, cereals, and beverages). Various phenolic compounds possess a diverse range of beneficial biological activities. Extensive research has been conducted in vitro or in vivo on antioxidant and anticancer activities of phenolic compounds from medicinal herbs and dietary plants. Phytochemicals are defined as bioactive nonessential nutrients from plants. Phenolic compounds provide essential functions in the reproduction and growth of plants; act as defense mechanisms against pathogens, parasites, and predators; as well as contribute to the color of plants [1]. Many phenolic compounds have been reported to possess potent antioxidant activity and to have anticancer, antibacterial, antiviral and anti-inflammatory activities $[2,3]$.

Phenolic compounds possess one or more aromatic rings bearing one or more hydroxyl groups with over 8,000 structural variants and generally are categorized as phenolic acids and analogs, flavonoids, tannins, stilbenes, curcuminoids, coumarins, lignans, quinones, and others based on the number of phenolic rings and of the structural elements that link these rings [4].

Recently, much attention has been focused on the macroalgae as sources of novel biologically active compounds such as phycobiline, phenols, terpenoids, steroids and polysaccharide $[5,6]$. Algal phenolic compounds were reported to be a potential candidate to destroy free radicals, which are harmful to our body and food systems [7]. Freshwater algae are a rich source of structurally novel and biologically active metabolites. In algae, there are antioxidant substances of very different nature. The most powerful water-soluble antioxidants found in algae are polyphenols, phenolic and vitamins.

Spirogyra is a genus of filamentous green algae of the order Zygnematales. It is commonly found in freshwater areas and there are more than 400 species of Spirogyra in the world. Spirogyra sp. (Division Chlorophyta) is an edible freshwater macroalga, consumed as traditional food in the north and northeast of Thailand due to its high nutrient content including vitamins, minerals and phenolic content [8]. The alga contains phenolic compounds which are the substances associated with biological activity [9]. The aqueous extracts of Spirogyra sp. exhibited in rats pharmacological effects, such as an antioxidant, anti-gastric ulcer, anti-inflammatory, antimutagenic and anti-diabetic activities, which suggested their value as therapeutic agents.

Cladophora glomerata has been naturally grown in the north of Thailand. It is commonly known in Thai as "Kai." Local people have traditionally used this alga as an ingredient in several northern Thai dishes and believed that it could serve as a medicinal plant for several diseases [10]. Previous in vivo studies have suggested that Cladophora glomerata extract exhibited antigastric ulcer, anti-inflammatory, analgesic, hypotensive and antioxidant activities in vitro and in vivo [10]. Phytochemical extraction is usually obtained by solidliquid extraction with different solvents. In research involving plants, various extraction methods have been developed for the extraction of phytochemicals in order

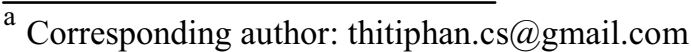


to increase the extraction yield, shorten the extraction time as well as enhance the quality of extracts.

Ultrasound-assisted extraction (UAE) is among the methods used to enhance the process of extraction. It is inexpensive, simple and efficient alternative to conventional extraction techniques. Like soxhlet extraction, it can be used with any solvent for extracting a wide variety of natural compounds. In solid-liquid extraction, it offers advantages which include increased yield and faster kinetics. The apparatus is cheaper and easier to operate compared to other novel extraction techniques such as microwave-assisted extraction and supercritical fluid extraction [11]. UAE has been proposed as a timid alternative to conventional solvent extraction, providing higher recovery of targeted compounds with lower solvent consumption and/or faster analysis and bioactivity properties. Its better extraction efficiency is related to the phenomenon called acoustic cavitation. When the ultrasound intensity is sufficient, the expansion cycle can create cavities or microbubbles in the liquid. Once formed, bubbles will absorb the energy from the sound waves and grow during the expansion cycles and recompress during the compression cycle. Further, bubbles may start another rarefaction cycle or collapse leading shock waves of extreme conditions of pressure and temperature [12,13]. Thus, the implosion of cavitation bubbles can hit the surface of the solid matrix and disintegrate the cells causing the release of the desired compounds. Nowadays, UAE is extensively used for the extraction of valuable molecules. For example, it has been used for the extraction of proteins [14], sugars [15], polysaccharides-protein complex [16], oil [17], and so on. However, the extraction of antioxidants such as phenolic compounds has been specially addressed optimizing their recovery based on its yield and antioxidant capacity by means of experimental design. These studies have showed that the recovery, antioxidant capacity and profile are strongly influenced by extraction variables where extraction time, temperature and frequency are the most important [18-20]. Some aspects related to the stability of the compounds extracted has not fully addressed, however, recent studies revealed that UAE of phenolic compounds were less degraded than others [21] and even no degradation has been observed under optimized conditions [22]. UAE was widely used in the extraction of plant compounds. However, no information is available on the effect of ultrasoundassisted extraction on polyphenol content in two freshwater macroalgae; Cladophora glomerata and Spirogyra sp. Hence, the objective of the present study was to probe the the usage of ultrasound in overcoming the limitation of conventional extraction of macroalgae as well as its effect on the phenolic content of two freshwater macroalgae.

\section{Materials and methods}

All chemicals were of analytical grade and purchased from Merck and Sigma-Aldrich. Solvents were used without further purification. Fresh Spirogyra sp. and Cladophora glomerata was purchased from a local supplier in Thailand and then washed and stored at $4 \pm 0.5^{\circ} \mathrm{C}$ at refrigerator for about one day for equilibration of moisture. The plant material was botanically identified. The collected algae were cleaned and dried parts were removed. Then the samples were rinsed with sterile water to remove any associated debris and dried at $35 \pm 2{ }^{\circ} \mathrm{C}$ in a microprocessor control drying oven. The dried algae were ground to fine powder using the commercial blender and kept at $-40^{\circ} \mathrm{C}$ until further use.

One gram of powdered sample was extracted in $80 \%$ ethanol where ratio of solid/liquid were at 1/20,1/30 and $1 / 40$ (wt/vol). Extraction was carried out at $400 \mathrm{rpm}$ at the temperature of $30^{\circ} \mathrm{C}$ and $40^{\circ} \mathrm{C}$ for 2 hours using shakers. The extracts were then filtered and analyzed for total phenolic content. The solid/liquid ratio and temperature which gave better result was selected and applied in ultrasonic-assisted extraction.

One gram of powdered sample in $80 \%$ ethanol was ultrasonicated for the duration of $5,10,15$ and $20 \mathrm{~min}$ using a $37 \mathrm{kHz}$ ultrasonic generator (VGT1990QTD). The mixture was then centrifuged at $400 \mathrm{rpm}$ for $30 \mathrm{~min}$ using Labofuge200, Thermo scientific. The extracts were filtered and used for further analysis.

Total phenolics were determined using a modified Folin-Ciocalteau colorimetric method [23,24]. A volume of $0.25 \mathrm{ml}$ of ethanolic extract was mixed with $1 \mathrm{ml}$ distilled water in a test tube. The $0.25 \mathrm{ml}$ of FolinCiocalteau reagent was added to the solution and allowed to react for $6 \mathrm{~min}$. Then, $2.5 \mathrm{ml}$ of $7 \%$ sodium carbonate solution was added into the test tubes, and the mixture was diluted to $6 \mathrm{ml}$ with deionized water. Each sample was allowed to stand for 90 minutes, and the absorbance measured at $760 \mathrm{~nm}$ using UV-Vis spectrophotometer. The measurement was compared to a standard curve of gallic acid concentrations and expressed as milligrams of gallic acid equivalents (GAE) per $100 \mathrm{~g}$ dried sample. The extractions and all analyses were executed at least in triplicate and data presentation was expressed as means and standard deviation.

\section{Results}

\subsection{Total phenolic content}

In determining total phenolic content of two freshwater macroalgae, dried material was prepared in this study. The total phenolic contents of two freshwater macroalgae were estimated using the Folin-Ciocalteu method, which relies on the transfer of electrons from phenolic compounds to the Folin-Ciocalteu reagent in alkaline medium. As recorded in Table 1, solid/liquid (wt/vol) ratio that gave a higher total phenolic content (TPC) reading was $1 / 30$ at $40^{\circ} \mathrm{C}$ used in two algae. Among three temperatures, TPC of two algae had the highest value at the temperature of $40^{\circ} \mathrm{C}$ for all solid/liquid ratios. Thus, it was concluded that TPC of two algae was highest at solid/liquid ratio of $1 / 30$ and at the temperature of $40^{\circ} \mathrm{C}$. This condition gave the best result and it was taken as the best condition to perform in further extraction. 
ICMCE 2015

Table 1 Total phenolic content of Spirogyra sp. and Cladophora glomerata without UAE.

\begin{tabular}{|c|c|c|c|}
\hline \multicolumn{4}{|c|}{ Temperature } \\
\hline & $30^{\circ} \mathrm{C}$ & $40^{\circ} \mathrm{C}$ & $\mathbf{5 0}^{\circ} \mathrm{C}$ \\
\hline Wt/Vol (g/ml) & mg GAE \pm SD & mg GAE \pm SD & mg GAE \pm SD \\
\hline \multicolumn{4}{|c|}{ Spirogyra sp. } \\
\hline $1 / 30$ & $1,684.52 \pm 1.43$ & $1,799.14 \pm 0.56$ & $1,730.33 \pm 2.26$ \\
\hline $1 / 40$ & $1,584.32 \pm 2.26$ & $1,739.22 \pm 0.98$ & $1,655.12 \pm 1.43$ \\
\hline $1 / 50$ & $1,358.81 \pm 0.85$ & $1,590.45 \pm 0.85$ & $1,489.21 \pm 0.44$ \\
\hline \multicolumn{4}{|c|}{ Cladophora glomerata } \\
\hline $1 / 30$ & $1,799.24 \pm 0.56$ & $1,989.47 \pm 0.28$ & $1,854.43 \pm 0.85$ \\
\hline $1 / 40$ & $1,743.32 \pm 0.28$ & $1,839.42 \pm 0.56$ & $1,801.59 \pm 2.26$ \\
\hline $1 / 50$ & $1,621.42 \pm 0.17$ & $1,890.22 \pm 1.43$ & $1,743.82 \pm 0.56$ \\
\hline
\end{tabular}

The results showed that the solvent ratio had an effect on the extraction and phenolic contents (Table 1). The best solvent ratio that yielded the highest phenolic content in Spirogyra $s p$. was $1 / 30 \mathrm{wt} / \mathrm{vol}$, followed by $1 / 40$ and $1 / 50 \mathrm{wt} / \mathrm{vol}$, respectively. For Cladophora glomerata, the sequence of solvent ratios that yielded the highest phenolic content was $1 / 30 \mathrm{wt} / \mathrm{vol}$, followed by $1 / 50$ and $1 / 40 \mathrm{wt} / \mathrm{vol}$, respectively. The results showed that the concentration of phenols grows logically while the ratio increases only in Spirogyra $s p$.

\subsection{Effect of UAE on total phenolic content}

Generally, the temperature has a positive effect on the extraction of phenolic compounds from plant and algae. The observed positive effect of temperature could be explained by the higher solubility of polyphenols in the solvent, the higher diffusivities of the extracted molecules and the improved mass transfer at higher temperatures. Although a higher temperature increased cavitation of ultrasonic assisted extraction by assisting in cell wall breaking in order to release the polyphenols, a higher temperature decompose phenolic compounds. The effect of heating temperature to the polyphenols extraction from plant material was associated to the types and different bound forms of polyphenols that are presented in material such as plants and algae depending on the species. Hence, different algae or plants resulted in different optimum extraction and recovery of phenolic content. For these results demonstrated that the temperature has a positive effect on the phenolic contents of algae extracts. The obtained results show also a very clear influence of the medium temperature on the phenolic content extraction. The phenolic contents of two extracts decreased following the sequence: extracts at $40{ }^{\circ} \mathrm{C}>$ extract at $50{ }^{\circ} \mathrm{C}>$ extract at $30^{\circ} \mathrm{C}$. (Table 1 ).

As indicated in Table 2, when extraction was repeated using UAE method, the TPC readings of two algae were higher compared to the extraction without UAE $(1,799.14$ $\mathrm{mg} \mathrm{GAE} / 100 \mathrm{~g}$ dried sample extracted using $1 / 30$ ratio at $40^{\circ} \mathrm{C}$ in Spirogyra $s p$. and $1,989.47 \mathrm{mg} \mathrm{GAE} / 100 \mathrm{~g}$ dried sample extracted using $1 / 30$ ratio at $40^{\circ} \mathrm{C}$ in Cladophora glomerata). The increasing in TPC of Spirogyra sp. and Cladophora glomerata was at $23 \%-37 \%$ and $\sim 27 \%-$ $39 \%$, respectively when 5 to $20 \mathrm{~min}$ of ultrasonication was applied.
Table 2 Total phenolic content for UAE of Spirogyra sp. and Cladophora glomerata with different ultrasonication duration.

\begin{tabular}{|c|r|}
\hline Time & mg GAE \pm SD \\
\hline & Spirogyra sp. \\
\hline $5 \mathrm{~min}$ & $2,215.81 \pm 0.20$ \\
$10 \mathrm{~min}$ & $2,384.21 \pm 1.22$ \\
$15 \mathrm{~min}$ & $2,468.13 \pm 0.33$ \\
$20 \mathrm{~min}$ & $2,341.92 \pm 1.31$ \\
\hline \multicolumn{2}{|c|}{ Cladophora glomerata } \\
\hline $5 \mathrm{~min}$ & $2,610.73 \pm 3.41$ \\
$10 \mathrm{~min}$ & $2,755.23 \pm 0.20$ \\
$15 \mathrm{~min}$ & $2,655.23 \pm 2.61$ \\
$20 \mathrm{~min}$ & $2,534.10 \pm 0.17$ \\
\hline
\end{tabular}

Among the four different durations used, the duration of $15 \mathrm{~min}$ gave the highest TPC reading at $2,468.13 \mathrm{mg}$ GAE/100g dried sample of Spirogyra sp. For Cladophora glomerata, the duration of $10 \mathrm{~min}$ gave the highest TPC reading at $2,755.23 \mathrm{mg} \mathrm{GAE} / 100 \mathrm{~g}$ dried sample. These TPC value were significantly higher when compared to the other duration.

The total phenolic content of all algae samples increased steadily as a function of time. There was a tendency to drop of the phenolic content after $20 \mathrm{~min}$ in Spirogyra sp. and after $10 \mathrm{~min}$ in Cladophora glomerata. This can be explained by the heating effect of overexposure to ultrasound treatment for longer extraction time, which leads to the degradation of phenolic contents of the extracts. These effects may be accordance with an antioxidant capacity of extracts.

\section{Discussion}

Bioactive compounds are sensitive to extraction techniques based on heat or solvent use. In addition, these techniques are time-consuming and energy intensive. It is necessary to identify and develop new efficient extraction

processes to utilize the bioactive present in macroalgae. To this end, researchers have been working toward the development of novel techniques that are more efficient in terms of yield, time, cost, in addition, are environmentally friendly. Many extraction technologies such as enzyme-assisted extraction (EAE), microwave-assisted extraction (MAE) and UAE have been successfully used in food and pharmaceutical 
applications for extraction of bioactive compounds. This work describes using UAE extraction technique that can be employed for the extraction of a range of phenolic content which was one of the bioactive compounds.

UAE is a simple, cost-effective, and efficient alternative to traditional extraction techniques. Benefits of using UAE include an increase of extraction yield and faster kinetics. Ultrasound facilitates the extraction of heat sensitive compound with minimal damage. It may also be used with a wide variety of solvents including the aqueous extraction of bioactive, that is, for water-soluble components [11]. The extraction mechanism of the UAE process occurs mainly in two stages, namely (1) dissolution of soluble components on surface of the algae matrix occurs which is known as "washing" and (2) mass transfer of the solute from the algae matrix into the solvent occurs by diffusion and osmotic processes which is known as "slow extraction"

In this study, the usage of ultrasound has successfully enhanced the extraction process as indicated by the significantly higher TPC values and furthermore, has reduced the centrifuge time from 2 hours to 30 minutes. This is made possible due to the propagation of ultrasound pressure waves through the solvent resulting in cavitation phenomena. The controlling mechanism of ultrasound-assisted extraction is generally attributed to mechanical, cavitation, and thermal effects which can result in disruption of cell walls, particle size reduction, and enhanced mass transfer across cell membranes, which lead to target compounds dissolving in the solvent, hence increasing yield with shorter time [25]. The TPC value of two algae using UAE was higher than that of Spirogyra sp. aqueous extract reported by Rattanapot [8] and Cladophora glomerata extract reported by Peerapornpisal [10]. This is possible from the advantage of UAE to extract the bioactive compounds.

The identified optimized processing conditions of extraction from this study will facilitate yield optimization of bioactive compounds from other macroalgae for industrial applications. Macroalgae processors require new novel extraction technologies with enhanced extraction yield and efficiencies compared to conventional solid liquid extraction methods. The results presented demonstrate that UAE has the potential technique to enhance the TPCs. The ultrasound technology is relatively cheap technology. It is also consumes less time for extraction. Simplicity of set up of equipment makes UAE one of the most suitable technologies for industrial extraction of phenolic and antioxidant compounds.

UAE has also been successfully used for extraction of many bioactive compounds including phenolic compounds, fucose and uronic acids. Most of results showed that this method gave higher phenolic content than the solid-liquid extraction method. Many solvents were used for extraction of phenolic from plants and algae such as ethanol, ethyl acetate, water and $\mathrm{HCl}$, respectively. It was concluded that ultrasonic extracts of two macroalgae of hold considerable potential against free radical toxicity by virtue of their polyphenolic constituents, and might have significant clinical roles in prospect.

\section{Conclusion}

The TPCs of two macroalgae extracted with and without the application of ultrasonic were compared in this study. The results clearly indicated that the values of TPC were significantly higher when the extraction procedure included ultrasonic as assistance in the method. These studies showed that phenolic compound extraction can benefit from UAE especially by reducing the extraction time. UAE is an interesting process to obtain high valuable compounds and could contribute to the increase in the value of some food byproducts when used as sources of natural compounds. The main benefits will be a more effective extraction, thus saving energy, and also the use of moderate temperatures, which is beneficial for heat sensitive compound. For a successful application of the UAE, it is necessary to consider the influence of several process variables, the main ones being the applied ultrasonic power, the frequency, the extraction temperature, the reactor characteristics and the solventsample interaction.

\section{Acknowledgement}

This work was supported from Faculty of Science, Department of Chemistry, Applied Chemistry, Maejo University and NRCT.

\section{References}

1. X.Z. Han, T. Shen and H.X. Lou, Int. J. Mol. Sci 8, 950-988 (2007)

S. Veeriah S, T. Kautenburger, N. Habermann, J. Sauer, H. Dietrich, Mol. Carcinogen 45, 164-174 (2006)

3. R.W. Owen, A. Giacosa, W.E. Hull, R. Haubner, B. Spiegelhalder, Eur. J. Cancer 36, 1235-1247 (2000)

4. P. Fresco, F. Borges, C. Diniz, and MPM. Marques, Med. Res. Rev 26, 747-766 (2006)

5. AH. Li, K. Cheng, C. Wong, F. King-Wai, C. Feng, J. Yue, Food. Chem 102, 771-776 (2007)

6. HH. Abd El-Baky, FK. El Baz, GS. El-Baroty, Eurasian J. Agric. Environ.Sci 3, 434-444 (2008)

Am.

7. JEP. Estrada, Bescos P. Bermejo, AM. Villar del Fresno, Farmaco 56, 497-500 (2001)

8. T. Rattanapot, K. Mengumphan, C. Srimaroeng, R. Junthip and D. Amornlerdpison, J. Fish. Tech. Res 6(2), 23-34 (2012)

9. N. Lailerd, A. Pongchaidecha, D. Amornlerdpison and Y. Peerapornpisal, Ann. Nutr. Metab 55(S1), 609 (2009).

10. Y. Peerapornpisal, D. Amornledpison, C. Rujjanawate, K. Ruangrit, and D. Kanjanapothi, Sci. Asia 32(1), 71-76 (2006)

11. L. Wang and C.L. Weller, Trends. Food. Sci. Technol 17(6), 300-312 (2006)

12. AC. Soria, M. Villamiel, Trends Food. Sci. Technol 21(7), 323-331 (2010)

13. M. Esclapez, J. Garc'ia-P'erez, A. Mulet, J. C'arcel, Food. Eng. Rev 3, 108-120 (2011)

14. W. Qu, H. Ma, J, Jia, R. He, L. Luo, Z. Pan, Ultrason Sonochem 19(5), 1021-1026 (2012)

15. B. Karki, BP. Lamsal, S. Jung, JH. Van Leeuwen, AL. Pometto, D. Grewell, SK. Khanal, J. Food. Eng 96(2), 270-278 (2010) 
16. YC. Cheung, KC. Siu, YS. Liu, JY. Wu, Process. Biochem 47(5), 892-895 (2012)

17. F. Adam, M. Abert-Vian, G. Peltier, F. Chemat, Bioresour. Technol 114, 457-465 (2012)

18. YC. Chukwumah, LT. Walker, M. Verghese, S. Ogutu, Ultrason. Sonochem 16(2), 293-299 (2009)

19. K. Ghafoor, YH. Choi, JY. Jeon, IH. Jo, J. Agric. Food. Chem 57(11), 4988-4994 (2009)

20. MB. Hossain, NP. Brunton, A. Patras, B. Tiwari, C. O’Donnell, AB. Martin-Diana, C. Barry-Ryan, Ultrason. Sonochem 19, 582-90 (2011)
21. P. Dobias, P. Pavlikova, M. Adam, A. Eisner, B. Benova, K. Ventura, Cent. Eur. J. Chem 8(1), 87-95 (2010) 22. D. Pingret, AS. Fabiano-Tixier, CL. Bourvellec, CMGC. Renard, F. Chemat, J. Food. Eng 11(1), 73-81 (2012)

22. A.L. Waterhouse, "Determination of Total Phenolics," in Current Protocols in Food Analytical Chemistry, (Wiley, I1.1.1-I1.1.8 2002)

23. K.L. Wolfe, X. Kang, X. He, M. Dong, Q. Zhang and R.H. Liu, J. Agric. Food. Chem 56(18), 8418-8426 (2008)

24. S.R. Shirsath, S.H. Sonawane and P.R. Gogate, Chem. Eng. Process. Intensif 53, 10-23 (2012) 